\title{
Doppler Shift Estimation and Compensation in High Speed MIMO-OFDM VANETS
}

\author{
Ferdinand C. Nyongesa ${ }^{1}$ and Thomas O. Olwal ${ }^{2}$ \\ ${ }^{1}$ Masinde Muliro University of Science and Technology, Kakamega, Kenya \\ ${ }^{2}$ Department of Electrical Engineering, Tshwane University of Technology, Pretoria, South Africa
}

\begin{abstract}
Motions in vehicular ad hoc networks (VANETs) cause Doppler Frequency Shifts (DFSs) which are described by frequency dispersions in the received signals. Although the VANETs channels are perturbed by noise sources such as multipath delay spread, shadowing and interference, a DFS due to the high speed vehicular mobility is a major cause of high bit-error rates (BER). Schemes for estimating and compensating DFSs in VANETs with medium to high speed mobility have been reported in the recent literature. However, significant computational complexity with some methods configured in MIMO-OFDM systems has rendered them non-attractive in full-scale deployment of high speed VANETs. The research question that remains unanswered is what scheme can be proposed to estimate and compensate for the DFS at a reduced computational complexity when MIMO-OFDM systems and high speed mobility conditions are considered? This paper answers this question by proposing a pilot-assisted DFS estimator and compensator based on the general complex exponential basis expansion matrix (BEM) modelling. The proposed method realizes a low computational complexity DFS estimator and compensator in the linear order of the Fast Fourier Transform (FFT) size as compared to the DFS uncompensated MIMO-OFDM systems whose computational complexity has been noted to be in a quadratic order of the FFT size. Numerical results indicate that the proposed BEM method demonstrates a better BER performance than the conventional approaches under the same MIMO configurations for moderately high vehicular speeds.
\end{abstract}

ACM CCS (2012) Classification: Networks $\rightarrow$ Network algorithms $\rightarrow$ Basis expansion modelling algorithms $\rightarrow$ Network design and planning algorithms

Networks $\rightarrow$ Network types $\rightarrow$ Ad hoc networks $\rightarrow$ Mobile ad hoc networks $\rightarrow$ Vehicular ad hoc networks Networks $\rightarrow$ Network performance evaluation $\rightarrow$ Network simulations
Keywords: basis expansion matrix modelling (BEM), doppler frequency shifts (DFS), inter carrier interference (ICI), multiple input multiple output (MIMO) systems, orthogonal frequency division multiplex (OFDM) systems, signal to noise ratio (SNR), vehicular ad hoc networks (VANETs)

\section{Introduction}

In vehicular ad hoc networks (VANETs), a high vehicular speed of the transmitting terminal renders itself to the effects of Doppler Frequency Shifts (DFS) which simply means the frequency dispersions in the received signal. DFS estimation and compensation significantly improves the quality of the received signal [1]. DFS estimation exploits the knowledge of fading rates of a channel which depends on its maximum DFS. Recently, Intelligent Transportation System (ITS) applications which promise safety and comfort of drivers on the roads have attracted attention of many researchers. The emergence of the ITS applications have witnessed an increased research interest toward estimating and compensating the DFS in low to medium speed (typically, below 120 $\mathrm{km} / \mathrm{h}$ ) VANETs [2]. As such, several methods have been proposed including the Error Vector Magnitude (EVM) methods [2], [26] and the one-tap split phasor least mean square (LMS) algorithm [3]. The latter isolates DFS from the received signals at different vehicular speeds. In [4], a DFS estimator based on level-crossing rate (LCR) is proposed, while the DFS es- 
timator based on an autocorrelation function $(\mathrm{ACF})$ is proposed in [5], [6], [7]. An extension to multiple input multiple output (MIMO) systems was investigated in [8], [25] and a frequency-domain DFS estimation method based on polynomial approximation to the zero order Bessel function of the first kind was proposed in [9]. In [10], the DFS in underwater acoustic (UWA) channel is extracted by time expansion and compression. The DFS was compensated via an efficient 4-th order Lagrange interpolation. In [11], the Doppler spread estimation is accomplished by determining the time autocorrelation function (TACF), which exploits pilot tones transmitted to the receiver.

Due to the highly dispersive nature of the vehicular channel, the channel matrix is a full matrix with time-varying (TV) coefficients caused by multi-path fading and Doppler spread. For time-invariant (TI) fading channels, the channel matrix in the frequency domain would be diagonal such that each sub carrier in the orthogonal frequency division multiplexed (OFDM) system is simply attenuated by the corresponding frequency domain channel response. In this case, a simple pilot-based channel estimator using interpolation may be adopted to estimate the channel in the frequency domain [12]. However, for rapidly varying channels, such as those under large Delay spread and Doppler spread, the variation of the channel within one OFDM symbol destroys the orthogonality between the sub carriers and this introduces non-zero off diagonal terms in the channel matrix constituting inter-carrier interference (ICI) [13]. In this case, an estimation of the channel variation in the frequency domain becomes complex due to the large number of unknowns in the channel matrix.

Hua et al. [4], proposed an estimation of DFS using an adaptive level-crossing rate (LCR) method. Hua et al. [4] exploited the proportionality relation between the LCR of the received signal envelope and the DFS to estimate the DFS. However, the authors did not take into consideration effects of the multi-path delay spread on DFS estimates from the envelope of the received signal fading rate. Moreover, a computationally complex iterative LCR approach was proposed without considering the high capacity MIMO channels [4]. The three issues such as multi-path delay spread, high computational system complexity and low capacity in the SISO channel render the algorithms in [4] unattractive in full scale deployment of VANETs in relatively high speed MIMO-OFDM systems [24].

It is imperative to note that the preceding discussions show a need to achieve a low computational complexity of DFS estimation and compensation methods. This study thus, has been compelled by the need to propose a pilot-assisted general complex exponential basis expansion modeling (BEM) technique to the DFS estimation and compensation in high speed MIMO-OFDM VANETs. The design and analysis in the paper begin with applying an OFDM system to the communication channel in order to combat effects of multi-path delay spread and delink such delay spread from the DFS. The paper then proposes the pilot assisted BEM method to combat inter-carrier interference (ICI). The paper contributes to the existing approaches on the application of the moderately high speed MIMO-OFDM VANET by modeling low computational complexity based DFS estimation and compensation algorithm. Also, the study analytically demonstrates that the proposed method is less computationally complex in relation to the conventional methods.

The rest of the paper is organized as follows. The system model is described in Section 2. The DFS estimator and compensation algorithm is outlined in Section 3. Performance evaluation and complexity analysis are presented in Sections 4 and 5. Section 6 concludes the paper.

The notations used in this paper are as follows: $x($.$) and x[$.$] denote continuous and discrete$ time-domain signals, respectively. The notations $\mathbf{x}$ [.] and $\mathbf{X}$ [.] denote vector and matrix functions, respectively.

\section{System Model}

In this section, let's consider a MIMO-OFDM based channel modelling of the time and frequency selective fading characteristics for high mobility conditions. Suppose an OFDM communication system with $M$ subcarriers in a MIMO configuration having $N_{t}$ transmit and receive antennas is represented by Figure 1. 


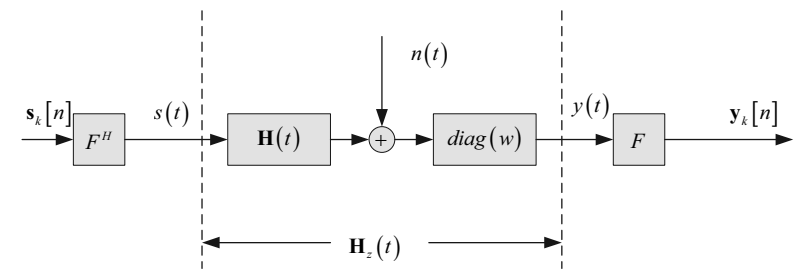

Figure 1. Communication transceiver and channel system.

The input-output relationship from Figure 1 may be expressed as

$$
\begin{aligned}
\mathbf{y}_{k}[n] & =F \operatorname{diag}(w)\left[\mathbf{H}(t) F^{H} \mathbf{s}_{k}[n]\right] \\
& +F \operatorname{diag}(w)[n(t)],
\end{aligned}
$$

where $F$ is the fast Fourier transform (FFT) operator, $\operatorname{diag}(w)$ is a windowed function designed to make the frequency-domain channel matrix as banded as possible, $\mathbf{H}(t)$ is the time-domain of the channel matrix of size $N_{t}$ $\times N_{r}, F^{H}$ is the inverse FFT operator, $\mathbf{s}_{k}[n]$ is the transmitted signal vector of the $k$-th OFDM symbol and the $n(t)$ is the additive white noise signal. By grouping the operation from (1), we have

$$
\begin{aligned}
\mathbf{y}_{k}[n] & =\underbrace{\left[\operatorname{Fdiag}(w) \mathbf{H}(t) F^{H}\right]}_{\mathbf{H}_{k}[n]} s_{k}[n] \\
& +\underbrace{\operatorname{Fdiag}(w)[n(t)]}_{\mathbf{z}_{k}[n]} \\
\mathbf{y}_{k}[n] & =\mathbf{H}_{k}[n] \mathbf{s}_{k}[n]+\mathbf{z}_{k}[n]
\end{aligned}
$$

Here, $\mathbf{H}_{k}[n]$ denotes the $N_{r} \times N_{t}$ frequency domain channel matrix of the MIMO system and $\mathbf{z}_{k}[k]$ represents the additive white Gaussian noise (AWGN) vector at the receiver. Each link channel $\mathbf{H}(t)$ between a pair of transmit and receive antennas can be regarded as an $L$-tap Finite Impulse Response (FIR) filter. The channel is assumed to be time varying (TV) over the transmission of every $k$-th OFDM symbol resulting from the multi-path delay spread and DFS fading effects. This means that $\mathbf{H}_{k}[n]$ channel matrix can be expressed as:

$$
\mathbf{H}_{k}[n]=\left[\begin{array}{cccc}
h_{1,1}[n] & h_{1,2}[n] & \cdots & h_{1, N_{r}}[n] \\
h_{2,1}[n] & h_{2,2}[n] & \cdots & h_{2, N_{r}}[n] \\
\vdots & \vdots & h_{i, j}[n] & \vdots \\
h_{N_{t}, 1}[n] & h_{N_{t}, 2}[n] & \cdots & h_{N_{t}, N_{r}}[n]
\end{array}\right]_{N_{t} \times N_{r}}
$$

where $h_{i, j}[n]$ stands for the TV channel impulse response (CIR) between the $j$-th $\left(j=1, \ldots, N_{r}\right)$ receive antenna and the $i$-th $\left(i=1, \ldots, N_{t}\right)$ transmit antenna when the $k$-th $(k=1, \ldots, K)$ OFDM symbol is being transmitted. The discrete complex baseband representation of a multi-path channel impulse response (CIR) of length $L$ can be described by the following [14], [15],

$$
h_{i, j}[n]=\sum_{l=0}^{L-1} A_{i, j}^{l}[n] \delta\left(t-\tau_{i, j}^{l}\right),
$$

where $\tau_{i, j}^{l}$ is the delay in the $l$-th path, normalized by the sampling period $T$, and $A_{i, j}^{l}[n]$ is the corresponding complex amplitude of the $k$-th symbol on the $l$-th path. We note from (4) that, due to the mobility of users, the sequences of $A_{i, j}^{l}[n]$ may be considered to be wide-sense stationary (WSS) narrowband complex Gaussian processes. Such complex amplitudes can also be assumed to be uncorrelated with each other in such a manner that each sample modulates an OFDM subcarrier. In OFDM modulation, the transmitter maps the message bits into a sequence of PSK or QAM symbols which will subsequently be converted into $M$ parallel streams. The interference between $M$ parallel data streams is eliminated by applying $M$ point inverse FFT to each block of $K$ data symbols at the transmitting station. Then, at the start of each $k$-th block, a cyclic prefix (CP) of length $M_{c p}$ is inserted as the guard interval to avoid inter symbol interference (ISI) [16]. This means that the $M_{c p}$ must be longer than the channel length to avoid ISI. The whole symbol duration $T_{\text {sym }}$ is taken to be $T_{\text {sym }}\left(M+M_{c p}\right) T$, where $M$ is the number of subcarriers in an OFDM symbol.

Thus, in discrete time, the baseband multicarrier signal radiated from the vehicular antenna is

$$
\mathbf{s}_{k}[n]=\sum_{m=0}^{M-1} \mathbf{s}_{k}[m] e^{j 2 \pi n k / M}, M_{c p} \leq m \leq M-1,
$$


where $M$ refers to the blocks of data symbols in an OFDM multicarrier system and $M_{c p}$ is the length of cyclic prefix. The $\mathbf{s}_{k}[n]$ is the $k$-th block, represented by the vector,

$$
\mathbf{s}_{k}=\left[\mathbf{s}_{k}(0), \mathbf{s}_{k}(1), \ldots, \mathbf{s}_{k}(M-1)\right]^{\tau}
$$

We can further assume that the vehicle moves at a speed of $v$ such that the DFS of the received signal will be given by

$$
f_{d}=\frac{f_{c} v}{c} \cos (\theta)=\frac{v}{\lambda_{c}} \cos (\theta) .
$$

where $f_{c}$ is the carrier frequency, $\lambda_{c}$ is the carrier wavelength of the received signal and $\theta$ denotes the angle between the direction of the moving vehicle receiving and the direction of the transmitting vehicle base station (BS). Then, the $k$-th OFDM symbol resulting from the DFS shifting effects are defined by

$$
\mathbf{s}_{k}^{D F S}[n]=\sum_{m=0}^{M-1} \mathbf{s}_{k}[m] e^{\left[j 2 \pi(m n / M)+j 2 \pi n f_{d} T_{s y m}\right]},
$$

where $T_{s y m}$ is the duration of the original data symbol. The above equation (7) shows that the DFS $f_{d}$ changes the OFDM symbol $\mathbf{s}_{k}^{D F S}[n]$ exponentially within the duration of the transmission of an OFDM symbol period. Consequently, the DFS deteriorates the performance of the OFDM system. When the $L$ resolvable paths in a multipath environment are assumed like in (4), then each path is parametrised by the direction of arrival (DoA) $\theta^{l}$, time delay $\tau^{l}$ (measured in unit of the symbol period $T_{\text {sym }}$ ), the DFS $f_{d}^{l}$ and complex path attenuation factor $\alpha_{k}^{l}$, which is assumed stationary within an OFDM symbol period, $T_{\text {sym }}$ but varying between OFDM symbols [16]. From equations (3) and (4) we have a channel impulse response equation:

$$
\mathbf{H}_{k}(t)=\sum_{l=0}^{L-1} a\left(\theta^{l}\right) \alpha_{k}^{l} e^{j 2 \pi f_{d}^{l} t} \delta\left(t-\tau^{l}\right),
$$

where $a\left(\theta^{l}\right)$ is the $N_{r} \times 1$ array response to a path from the direction $\theta^{l}$, which for the uniform linear array takes the form $a\left(\theta^{l}\right)=\left[1, e^{-j 2 \pi \frac{d}{\lambda_{c}} \sin \left(\theta^{l}\right)}, \ldots, e^{-j 2 \pi\left(N_{r}-1\right) \frac{d}{\lambda_{c}} \sin \left(\theta^{l}\right)}\right]^{T}$

Here, $d$ is the inter-element spacing of the antenna array. As the transmitted signal $\mathbf{s}_{k}[n]$ passes through the multi-path wireless channel defined by the impulse response $\mathbf{H}_{k}[t]$, the transmitted signal gets corrupted by a spatially uncorrelated additive white Gaussian noise $\mathbf{z}(t)$. The transmitted signal is corrupted by a number of situations, namely

(i) multi-path delay spread induced by the channel diversity,

(ii) Doppler spread caused by DFS, and

(iii) AWGN, $n(t)$.

The multi-path delay spread can be resolved by invoking the inverse FFT in the OFDM modulation process [17]. In OFDM modulation, a high-rate channel is demultiplexed into parallel low-rate sub channels which are robust to multi-path fading when the cyclic prefix (CP) is added [18]. In what follows, we first mitigate the multi-path delay, then estimate and compensate for the DFS effects.

\subsection{Mitigation of Multi-path Delay Spread Using OFDM technique}

Let's first mitigate the multi-path delay spread induced by the channel diversity by using the OFDM modulation technique proceeding to analyse the DFS effects due to the high mobility DFS of the vehicles. In the OFDM modulated communication systems, the multiplexed signal subcarriers can be described by $\left\{e^{j 2 \pi f_{m} t}\right\}_{m=0}^{M-1}$ which represents the different subcarriers at $f_{m}$ $=m / T_{\text {sym }}$, where $f_{m}$ is the frequency of the $m$-th subcarrier, $M$ denotes the number of ODFM subcarriers in the system and $T$ is the sample time interval denoted as $T=\frac{T_{s y m}}{M+M_{c p}}$. In the $k$-th OFDM block, the discrete-time received signal vector at the antenna array of the vehicle receiving station (BS) can be expressed as the convolution of the transmitted signal $\mathbf{s}_{k}[n]$ with the channel matrix $\mathbf{H}_{k}[n]$ as follows: 


$$
\begin{aligned}
\mathbf{y}_{k}[n] & =\sum_{l=1}^{L} \alpha_{k}^{l} \mathbf{a}\left(\theta^{l}\right) s_{k}^{D F S}\left[n-\tau^{l}\right]+\mathbf{z}[n] \\
& =\sum_{l=1}^{L}\left\{\alpha_{k}^{l} \mathbf{a}\left[\theta^{l}\right] e^{j 2 \pi\left[n-\tau^{l}\right] f_{d}^{l} T_{s y m}} \sum_{m=0}^{M-1}\left[s_{k}[m] e^{j 2 \pi\left[n-\tau^{l}\right] m / M}\right]\right\}+\mathbf{z}[n] \\
& =\sum_{m=0}^{M-1} \mathbf{s}_{k}[m] e^{j 2 \pi n m / M} \sum_{l=1}^{L}\left[\alpha_{k}^{l} \mathbf{a}\left[\theta^{l}\right] e^{j 2 \pi\left[n-\tau^{l}\right] f_{d}^{l} / M \Delta f} e^{-j 2 \pi \tau^{l} m / M}\right]+\mathbf{z}[n]
\end{aligned}
$$

where $\mathbf{y}_{k}[n]$ is the $n$-th sample of the antenna array output and $\mathbf{z}[n]$ is a $N_{r} \times 1$. A Gaussian noise vector with covariance matrix $\sigma^{2} \mathbf{I}_{N_{r}}\left(\mathbf{I}_{N_{r}}\right.$ is the $N_{r} \times N_{t}$ identity matrix) [16]. Let us define

$\mathbf{H}_{k, m}[n]=\sum_{l=1}^{L}\left[\alpha_{k}^{l} \mathbf{a}\left[\theta^{l}\right] e^{j 2 \pi\left[n-\tau^{l}\right] f_{d}^{l} / M \Delta f} e^{-j 2 \pi \tau^{l} m / M}\right]$.

At the receiver, the orthogonality conditions of the subcarriers at frequencies $f_{m}$ and $f_{i}$ must be satisfied for a given received signal $x(t)$ as follows:

$$
\begin{aligned}
x(t) & =\frac{1}{T} \int_{0}^{T} e^{j 2 \pi f_{m} t} e^{-j 2 \pi f_{i} t} d t \\
& =\frac{1}{T} \int_{0}^{T} e^{j 2 \pi\left(f_{m}-f_{i}\right) t} d t= \begin{cases}1, & \text { for } f_{m}=f_{i} \\
0, & \text { otherwise }\end{cases}
\end{aligned}
$$

with the equivalent discrete form expressed as [20]

$$
x[n]=\frac{1}{M} \sum_{m=0}^{M-1} e^{j 2 \pi\left(f_{m}-f_{i}\right) k}= \begin{cases}1, & \text { for } f_{m}=f_{i} \\ 0, & \text { otherwise }\end{cases}
$$

The above orthogonality condition is essential for the OFDM signal to be free from the effects of inter-carrier interference (ICI).

At the receiver, in the range $0 \leq n \leq M-1$ of the $k$-th OFDM symbol, the received signal is not corrupted by the previous OFDM symbols due to the presence of the cyclic prefix. Then, $\mathbf{y}_{k}[n]$ can be rewritten as:

$$
\begin{gathered}
\mathbf{y}_{k}[n]=\sum_{m=0}^{M-1} s_{k}[m] \mathbf{H}_{k, m}[n] e^{j 2 \pi n m / M}+\mathbf{z}[n] \\
0 \leq n \leq M-1 .
\end{gathered}
$$

The next step is to efficiently detect the desired information symbol

$$
\mathbf{s}_{k}=\left[s_{k}[0], s_{k}[1], \ldots, s_{k}[M-1]\right]^{T}
$$

from the DFS corrupted received signal $\mathbf{y}_{k}[n]$.

\subsection{Estimation and Compensation of Doppler Frequency Shift Using BEM Technique}

In the preceding section, the multipath interference has been eliminated using the OFDM orthogonality principle. In what follows, the major cause of the system performance degradation is attributed to DFS. The received signal suffers degradation on the channel arising from DFS as a result of high node mobility. Due to the arising Doppler spread, the channel matrix coefficients in (8) vary depending on the value of the Doppler spread. In our model, the channel is modelled with exponentially decaying path gains as a function of the DFS. Substituting $f_{d}$ in (11), so that each value of the coefficients in the channel matrix in (11) due to (4) is dependent on the speed of the moving vehicle $v$, the coefficient of the channel matrix becomes

$$
\hat{h}=h_{i, j} e^{-\left(f_{c}-f_{d}\right)^{-1}}=h_{i, j} e^{-\left(1-\frac{v}{c} \cos \theta\right)^{-1} f_{c}^{-1}},
$$

Normalizing (15) to $\exp \left(f_{c}^{-1}\right)$, the gains become

$$
\hat{h}_{\text {norm }}=h_{0} e^{-\left(1-\frac{v}{c} \cos \theta\right)^{-1}}
$$

where $h_{0}$ is a stationary gain. In the limits $v \rightarrow 0$,

$$
\hat{h}_{\text {norm }}=h_{0} e^{-\left(1-\frac{v}{c} \cos \theta\right)^{-1}} \rightarrow h_{0} e^{-1},
$$


constituting the well-known channel matrix with constant gains for the quasi-static channel. However, as $v \rightarrow c$, the $\hat{h}_{\text {norm }}$ may take the form:

$$
\begin{aligned}
\hat{h}_{\text {norm }} & =h_{0} e^{-\left(1-\frac{v}{c} \cos \theta\right)^{-1}} \rightarrow h_{0} e^{-(1-\cos \theta)^{-1}} \\
& = \begin{cases}h_{0} & \text { for } \theta=0^{0} \\
h_{0} e^{-1} & \text { for } \theta=90^{0}\end{cases}
\end{aligned}
$$

and as $v \rightarrow \infty$ then $\hat{h}_{\text {norm }}=h_{0} e^{-\left(1-\frac{v}{c} \cos \theta\right)^{-1}} \rightarrow 0$, implying that at extremely high vehicular speed the channel is extremely lossy so that it has no output $y(t)$ caused by extreme effects of mobility. In our model, $v$ is scaled by a constant $0 \leq \alpha \leq 1$ to define the realistic channel gains expressed as:

$$
\hat{h}_{\text {norm }}=h_{0} e^{-\left(1-\frac{\alpha v}{c}\right)^{-1}} .
$$

Under this model, a critical speed referred to as the High Speed (HS) will depict the extent of the speed of mobile vehicles beyond which DFS compensation will be performed. It is worth noting that in this paper, at HS, the symbol time and the channel coherence time are taken to be equal, beyond which the channel becomes fast fading and the signal becomes severely distorted. Thus, it can be shown that at $T_{s y m}=T_{c}$, the critical speed is represented as:

$$
v_{c}=\frac{c}{T_{c} f_{c}}=H S .
$$

Given that different channel models have different coherence time, it follows that HSs of such channels will similarly differ for a given signal. For successful decoding to be realized in channels beyond HS position, estimation and compensation of DFS are deemed necessary.

\subsection{Doppler Frequency Shift Estimation}

Consider a signal with baseband $B_{s}$ having symbol time $T_{\text {sym }}=1 / B_{s}$ transmitted over the channel. Defining $\sigma_{\tau}$ as the RMS delay spread, [14] gives the expression of the channel coherence bandwidth as:

$$
B_{c} \approx \frac{1}{\sigma_{\tau}} .
$$

Over this channel, frequency-selectivity is governed by signal bandwidth. Each path experiences a different amount of DFS such that the total frequency dispersion in the received signal is $\Delta_{f}$, the Doppler spread $B_{D}$. The reciprocal is the coherence time of the channel $T_{c}$, that is, $T_{c}$ $=1 / B_{D}$. When $T_{\text {sym }}>T_{c}$, then a fast fading occurs [19], it therefore follows that high mobility will increase the Doppler spread $B_{D}$. Hence, the fading speed of the channel can also be measured by the Doppler spread. Conversely, DFS is estimated as:

$$
B_{D} \approx \frac{1}{T_{c}}
$$

so that the rate of channel fading can estimate DFS experienced in that channel. However, (20) does not provide the explicit amount of DFS present sufficiently to enable compensation.

In frequency domain, at low vehicular speeds, $B_{D}$ is low and, as long as $B_{D}<B_{c}$, the channel is flat fading and the effect of DFS is not experienced. However, as speed increases, $B_{D}$ also increases according to (20). In the event that $B_{D}$ $>B_{c}$, the signal will undergo fast fading and be filtered by the channel. This will generate the out-of-band interference that will reduce signal quality. Consequently, the DFS-perturbed channel matrix from the coefficient matrix in (15)

$$
\hat{h}_{\text {norm }}=h_{0} e^{-\left(1-\frac{\alpha v}{c}\right)^{-1}},
$$

becomes

$$
\mathbf{H}_{k}^{D F S}=\mathbf{H}_{0} e^{-\left(1-\frac{\alpha v}{c} \cos \theta\right)^{-1}}
$$

so that the amount of DFS is estimated by the following models

$$
\begin{gathered}
e^{-\left(1-\frac{\alpha v}{c} \cos \theta\right)^{-1}} I_{N_{t} N_{r}}=\mathbf{H}^{-1} \mathbf{H}_{k}^{D F S} \\
\overleftrightarrow{\mathbf{H}}=\mathbf{H}^{-1} \mathbf{H}_{k}^{D F S}=e^{-\left(1-\frac{\alpha v}{c} \cos \theta\right)^{-1}} I_{N_{t} N_{r}} .
\end{gathered}
$$


In OFDM systems perturbed by DFS, most inter carrier interference (ICI) is concentrated in adjacent subcarriers, which implies that the channel matrix is roughly banded. The power is concentrated in the main diagonal in the channel matrix and falls off to zero away from the diagonal [20]. The BEM method provides a convenient approach to reduce interference due to DFS by nullifying out-of-band interference and simplifying computation by reducing the entries to be estimated. Under the BEM method, instead of estimating the entire channel matrix entries in $\mathbf{H}_{k}^{D F S}$, we basically estimate the BEM coefficients, by use of a matrix $\mathbf{B}$ of basis coefficients [18]. In BEM approach, information symbols $\mathbf{s}_{k}^{D F S}$ and the pilot sample noise $n^{(p)}$ in $\mathbf{H}_{k}^{D F S}$ are both zero-mean and uncorrelated with each other. The channel vector $\hat{h}[n]$ is considered random and uncorrelated to the two. A linear filter $W$ called a linear minimum mean square error (LMMSE) can then be chosen to minimize the mean square error (MSE) between the estimated and the true BEM coefficients [21].

$\hat{h}_{\text {LMMSE }}=R_{\hat{h}} \mathbf{s}_{k}^{H}[n]\left(\mathbf{s}_{k}[n] R_{\hat{h}} \mathbf{s}_{k}^{H}[n]+R_{I}\right)^{-1} \mathbf{y}_{k}^{(p)}[n]$,

where $\mathbf{s}_{k}[n]$ is the vector for all information samples derived in [18], [21]; $R_{\hat{h}}=E_{\hat{h}}\left\{\hat{\mathbf{h}} \hat{\mathbf{h}}^{H}\right\}$, $R_{I}=R_{d}+R_{n}^{(p)} \quad$ with $\quad R_{d}=E_{h, s}(d)\left\{d d^{H}\right\}$ and $R_{n}^{(p)}=E_{n}^{(p)}\left\{\mathbf{n}^{(p)} \mathbf{n}^{(p) H}\right\}$ being the autocorrelation coefficients and $E_{x}\{$.$\} is the expected value$ with respect to the random variable $x\{$.$\} . From$ (23), the MSE of LMMSE estimator is

$$
M S E_{\text {LMMSE }}=\operatorname{trace}\left\{\left(\mathbf{s}_{k}^{H}[n] R_{I}^{-1} \mathbf{s}_{k}[n]+R_{\hat{h}}^{-1}\right)^{-1}\right\} .
$$

In the BEM system, we assume a finite impulse response (FIR) channel with order $L$ as in (4). Collecting the time-variation of the $l$-th channel tap within the considered OFDM symbol in an $M \times 1$ vector $\mathbf{h}_{l}^{(t)}=\left[h_{M_{c p}, l}^{(t)}, \ldots, h_{M+M_{c p}-1, l}^{(t)}\right]^{T}$, we can express $\mathbf{h}_{l}^{(t)}$ as $\mathbf{h}_{l}^{(t)} \approx \mathbf{B} \mathbf{h}_{l}$, where $\hat{\mathbf{A}}=\left[b_{0}, \ldots, b_{Q}\right]$ is an $\mathbf{h}_{l}=\left[h_{0, l}, \ldots, h_{Q, l}\right]^{T}$ matrix that collects $M \times(Q+1)$ orthonormal basis functions $b_{q}$ as columns, and $\mathbf{h}_{l}=\left[h_{0, l}, \ldots, h_{Q, l}\right]^{T}$ with $h_{q, l}$ representing the $q$-th BEM coefficient for the $l$-th channel tap, so that the total number of estimated symbols is $(L+1)(Q+1)$ [21]. Consequently, the estimated matrix derived from (22) is expressed as:

$$
\overline{\mathbf{H}}_{k}=\mathbf{B H}_{k}^{-1} \mathbf{H}_{k}^{D F S}=\mathbf{B} e^{-\left(1-\frac{\alpha v}{c} \cos \theta\right)^{-1}} I_{N_{r} N_{t}},
$$

is a diagonal matrix of dimensions $(L+1)$ by $(Q+1)$ with eigenvalues representing the estimation error between the DFS-perturbed and actual coefficients. The processed $(L+1)(Q+$ 1) symbols are a reduction from the full matrix contents. This is a big saving in terms of computational resources such as memory space and processing time, compared to the conventional method which processes $M^{2}$ symbols, $M$ being the FFT size [2], [4], [12], [26]. Moreover, the zero off-diagonal entries cancel the out-of-band noise coefficients to enhance BER performance.

\subsection{Doppler Frequency Shift Compensation}

The objective of the DFS compensation is to nullify the factor

$$
\exp \left(-\left(1-\frac{\alpha v}{c} \cos \theta\right)^{-1}\right)
$$

in the matrix $\overline{\mathbf{H}}_{k}$ in (25) so that the unperturbed system could be restored. Consequently, the compensator is modelled as the product of the DFS-corrupted channel (22) and the inverted estimator (25) as:

$$
\overline{\mathbf{H}}_{k, \text { comp }}=\left(\mathbf{B H}_{k}^{-1} \mathbf{H}_{k}^{D F S}\right)^{\dagger} \mathbf{H}_{k}^{D F S}=I,
$$

where $(.)^{\dagger}$ is the pseudo-inverse. Finally, the DFS estimated and compensated signals are generated as:

$$
\hat{\mathbf{s}}_{k}[n]=\overline{\mathbf{H}}_{k, \operatorname{comp}}[n] \mathbf{H}_{k} \mathbf{y}_{k}[n]=\mathbf{H}_{k} \mathbf{y}_{k}[n] .
$$

The application of BEM operation leads to a less complex computation system due to the re- 
duction in the number of symbols and enhanced BER performance as a result of out-of-band interference cancellation due to application of BEM.

The noisy and DFS-impaired received signals $y(t)$ are demodulated in the carrier demodulator and converted back to the discrete domain by A / D conversion as a reverse process of the transmitter action. The discrete serial data stream is converted to $M$ parallel streams before CPs are removed, followed by FFT processing. The resulting frequency-domain parallel symbols are demapped into time-domain symbols and converted from parallel to serial data stream presented by (1) while pilots are recovered. The overall transceiver system is shown in Figure 2.

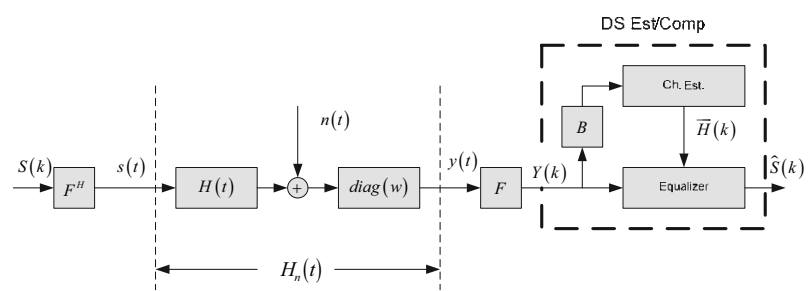

Figure 2. DFS compensated communication system.

The semi-processed received signal $\mathbf{y}_{k}[n]$ is split into two branches (Figure 2), one leading into the estimator via the basis coefficient matrix $\mathbf{B}$ and the other branch is directed to the compensator based equalizer. The general complex exponential BEM estimator characterizes the interference noise from the DFS and AWGN channels as one entity and cancels them altogether. The channel is equipped with a windowed function designed to make the frequency-domain channel matrix as banded as possible. The banding technique helps to identify out-of-band interference acquired on the channel which is caused by DFS. The basis coefficient matrix $\mathbf{B}$ is a diagonal matrix which must be designed to cancel the out-ofband interference to generate an error signal in the matrix $\overline{\mathbf{H}_{k}}[n]$ that estimates the amount of DFS. The product of its pseudo-inverse and the DFS-impaired channel matrix models the compensator which compensates for distortions in the direct signal. The output from the DFS Est / Comp block is the DFS-compensated signal $\hat{\mathbf{s}}_{k}[n]$ released to the detector for decoding.

\section{DFS Estimation and Compensation Algorithm Using Basis Expansion Modelling Matrix (BEM)}

The steps undertaken in our method to estimate and compensate DFS are summarised in the following pseudo code algorithm (Algorithm 1):

Algorithm 1. BEM: Basis expansion modelling based DFS stimation and compensation algorithm.

Objective: To estimate and compensate DFS in high speed MIMO-OFDM VANETs.

Step 1: Initialise

$f_{5}=0, T_{\text {sym }}=$ symbol period, $f_{S}=0, T_{S}=0, v=0$, $B_{D}=0, B_{c}=0, \sigma_{\tau}=0 ; M_{c p}=0, M=0$.

Step 2: Remove Multipath Fading

IFFT $\left(s_{n}\right) \& \&$ insert $M_{c p} ; \sigma_{\tau} ; / *$ insert CP; RMS delay spread $* /$

while $\left(T_{\text {sym }}<\sigma_{\tau}\right) / *$ multipath interference */ $M_{c p}=\left(M_{c p}+1\right) ; / *$ increase $\mathrm{CP}^{*} /$ go to step 2

else (continue);

Step 3: Test for DFS

if $(v \neq 0) / *$ mobility, Eqn. $17 * /$

do

$B_{c}=1 / \sigma_{\tau} ; B_{D}=1 / T_{c} ; / *$ Eqn. 19-20*/

if $\left(B_{D}<B_{c}\right) / *$ flat fading; low speed DS*/

go to step $6 ; / *$ decode signal ${ }^{*} /$

else if $(v>=\mathrm{HS}) / *$ fast fading; high speed DS present*/, Eqn. 19-20

(continue)

Step 4: Estimate DFS

compute

$\overrightarrow{\mathbf{H}}=\mathbf{B H}^{-1} \mathbf{H}^{D F S}$;

/*reduce the number of coefficients using BEM*/, Eqn. 25.

if $\overrightarrow{\mathbf{H}} \neq \mathbf{I}(v) ; / * \mathrm{DS}$ estimation, Eqn. $25 * /$

go to step 3

else (continue)

Step 5: Compensate DFS

$$
\begin{aligned}
& \overline{\mathbf{H}}_{\text {comp }}=\left(\mathbf{B} \mathbf{H}^{-1} \mathbf{H}^{D F S}\right)^{\dagger} \mathbf{H}^{D F S} ; \underset{\text { Eqn. } 26}{/ * \text { compensator*/, }} \\
& \hat{\mathbf{s}}_{k}[n]=\overline{\mathbf{H}}_{k, \text { comp }}[k] \mathbf{y}_{k}[n] ; / * \text { DS compensated } \\
& \text { (continue) }
\end{aligned}
$$

Step 6: Decode signal

$$
\begin{aligned}
& \text { end if } \\
& \text { end if } \\
& \text { end if } \\
& \text { end if } \\
& \text { end while } \\
& \text { end }
\end{aligned}
$$




\section{Performance Evaluation}

This section provides the performance evaluation of the pilot assisted general complex exponential basis expansion modelling (BEM) based DFS estimation and compensation method in a moderately high speed MIMO-OFDM VANET [22], [23]. The simulation was conducted with the number of sub-carriers set to be $M=1024$ and the length of the cyclic prefix (CP) was $M / 8$. Each transmitted packet contained one 50 millisecond chirp signal followed by 12.5 millisecond silent period. A total of 8920 information bits were transmitted in each setting. We used bandwidth $B=4 \mathrm{kHz}$, which led to a sub-carrier spacing of $3.90625 \mathrm{~Hz}$. The guard interval was set as $T_{g}=16$ millisecond. A rate $1 / 2$ non-systematic convolutional (NSC) code and interleaver were adopted in this simulation to map 892 data bits to 1792 interleaved bits. The achieved data rates were $3.2794 \mathrm{~kb} / \mathrm{s}$ and the bandwidth utilization factor was 0.8198 $\mathrm{bits} / \mathrm{sec} / \mathrm{Hz}$. We further considered a moderately high speed vehicle environment such that the scattering fading channels could validly be modelled as a six-tap $(L=6)$ Wide Sense Stationery (WSS) tap-delayed-line channel since the symbol rates were considered faster than the vehicular speeds. However, the channel had an exponential decay multi-path intensity profile with a time-varying property, i.e.,

$$
\sigma_{p}^{2}=\exp (-p / L) / \sum_{p=0}^{L-1} \sigma_{p}^{2}
$$

$p=0,1, \ldots, 5,[18]$. On another contribution and as an extension of this paper, we are currently investigating the effects of the non-isotropic scattering fading channels on the DFS estimation and compensation algorithms for extremely high vehicular speed greater than $150 \mathrm{~km} / \mathrm{h}$. We will be assuming von MISES angle of arrival distribution, having different Kappa factor, applied to generate directional fading channels [27].

In this sequel, the path gain for each channel tap is independently generated with the Jakes' Doppler spectrum containing maximum Doppler frequency $f_{d}$ [18]. The time interval between adjacent taps is $T_{\text {sym }} / M$, where $T_{\text {sym }}$ is one OFDM symbol duration. The associated DFS for every tap is distributed in $[-0.1 \Delta f, 0.1 \Delta f]$, where $\Delta f$ denotes the subcarrier interval. The performance simulation study is performed under two different normalized DFS frequencies, namely $f_{d}=0.2$ and $f_{d}=1$ corresponding to the absolute DFS of $334 \mathrm{~Hz}$ and $1632 \mathrm{~Hz}$ at $25 \mathrm{~dB}$ SNR environment. These DFS frequencies correspond to the vehicular speeds of $30 \mathrm{~km} / \mathrm{h}$ (or $8.3 \mathrm{~m} / \mathrm{s}$ ) for low vehicle speed regime and 150 $\mathrm{km} / \mathrm{h}(41.5 \mathrm{~m} / \mathrm{s})$ for high vehicle speed regime, respectively. The signal carrier frequency is taken at $5.9 \mathrm{GHz}$ with the number of transceiver antenna pairs $N_{t}$ and $N_{r}$ being 2 by 2 to 4 by 4 .The DFS estimation performance is then measured in terms of the Minimum Square Error (MSE) versus Signal to Noise Ratio (SNR).

Depending on the extent of the Doppler spread, the received signal undergoes fast or slow fading. For a transmission signal with symbol time $T_{\text {sym }}=3 \times 10^{-3}$ seconds, the critical speed $v_{c}$ is defined as shown in Figure 3.

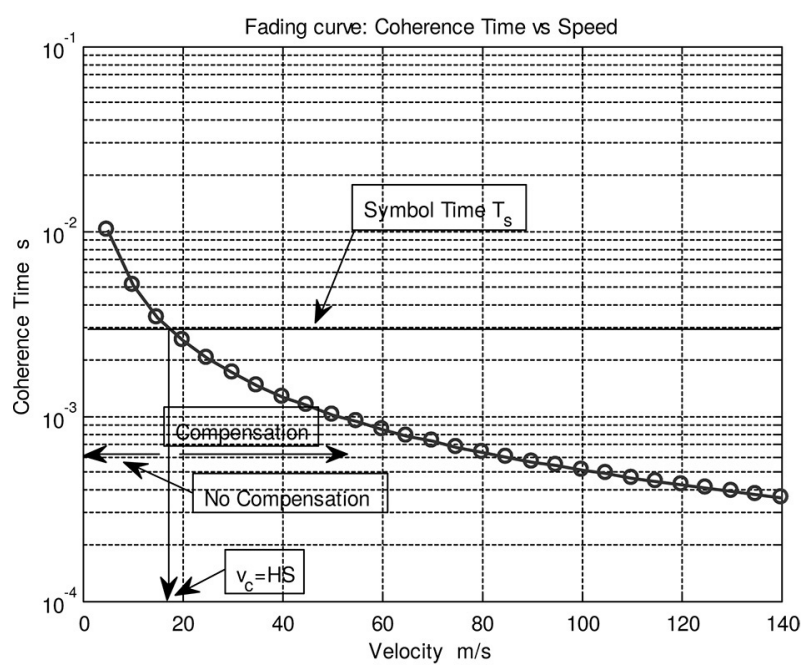

Figure 3. Coherence time variation with vehicular velocity.

As presented in Figure 3, the received signal undergoes slow fading up to a vehicular speed of $18 \mathrm{~m} / \mathrm{s}$ or $64.8 \mathrm{~km} / \mathrm{h}$. In this region, the signal requires no DFS compensation. However, as the vehicular speed increases further, the coherence time reduces and fast fading becomes dominant. This phenomenon requires that we compensate for the DFS for vehicular speed above $18 \mathrm{~m} / \mathrm{s}$. For the purpose of evaluating the Bit Error Rate (BER) versus Signal to Noise 
Ratio (SNR) performance of the proposed BEM against the traditional approaches, we denote this critical speed as HS.

In Figure 4, the system designed BER performance of the BEM algorithm for a $2 \times 2$ MIMO-OFDM VANET at different speeds has been shown. The reason for the performance evaluation is to ascertain whether the BEM system designs possess the ability to compensate the DFS offsets at refined individual symbol location. Thus, or a specific speed of the vehicle, the BER values are computed discretely at the receiver for every independently transmitted, recovered and DFS compensated $k$-th OFDM symbol denoted by $\hat{\mathbf{s}}_{k}[n]$ for the $n$-th sample of antenna array output. The compensated received signal vector of $K$ symbols is denoted as $\hat{\mathbf{S}}=\left[\hat{\mathbf{s}}_{1}, \hat{\mathbf{s}}_{2}, \ldots, \hat{\mathbf{s}}_{k}, \ldots, \hat{\mathbf{s}}_{K}\right]^{T}$. The received signal bit vector per symbol is counted and compared with the transmitted bit vector and the resulting error vector is divided by the length of the transmitted bit vector per symbol to obtain the BER values. Thus, due to randomness of the error vector, multiple simulation runs are performed at each symbol location, from the first to the 20th symbol, as illustrated in Figure 4.

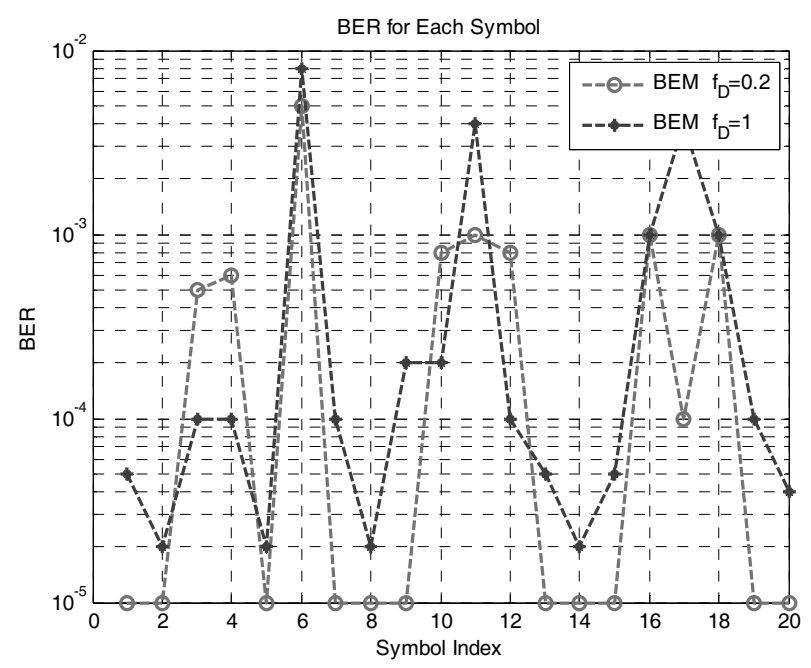

Figure 4. BER versus Symbol equaliser performance at $25 \mathrm{~dB}$ of the SNR in the low and high speed regimes.

The mean BER across OFDM symbols obtained in the highway environment in the low speed regime at $8.3 \mathrm{~m} / \mathrm{s}$ or $30 \mathrm{~km} / \mathrm{h}$ vehicle speed is $5.5 \times 10^{-4}$ whereas $9.6 \times 10^{-4}$ is obtained at
$41.7 \mathrm{~m} / \mathrm{s}$ or $150 \mathrm{~km} / \mathrm{h}$ for BEM method. This represents a $2.4 \mathrm{~dB}(74.5 \%)$ degradation in the BER performance due to the increased speed as compared to the $8.3 \mathrm{~m} / \mathrm{s}$ speed condition. The increased speed causes increased channel fading rate and increased DFS, thus lowering the performance at high speed. In Figure 5, the non-compensated [2], iterative LCR [4] and proposed BEM methods are compared in two vehicular speed regimes (below and above HS, respectively).

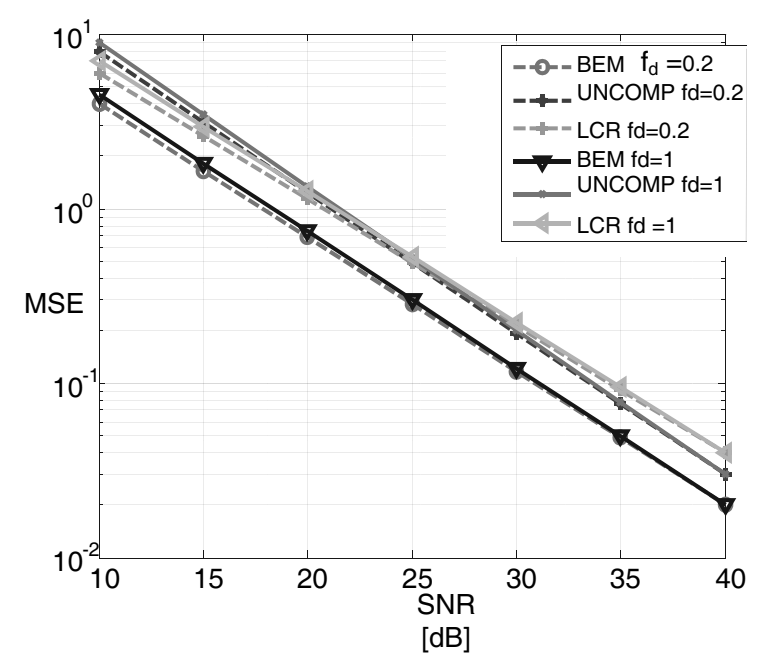

Figure 5. MSE versus SNR [dB] performance in two velocity regimes.

The comparative results in Figure 5 show that BEM mean square error (MSE) performance is generally better than that of LCR and non-compensated in different vehicular speed regimes. This is because the BEM method makes use of OFDM orthogonality principle to eliminate signal fading contributed by multi-path delay spread. In addition, the out-of-band noise interference is then cancelled by the basis coefficients in the channel matrix. In Figure 6, the BER performance of the BEM, LCR and uncompensated DFS estimation algorithms have been plotted against every $k$-th OFDM symbol denoted by $\hat{\mathbf{s}}_{k}[n]$ for the $n$-th sample of antenna array output. Like explained in Figure 4, the BER computations are performed after every symbol duration denoted as $T_{\text {sym }}$ in seconds.

The averaged BER performance measured for each symbol position in the received signal for the uncompensated system is $4.5 \times 10^{-2}$ as com- 


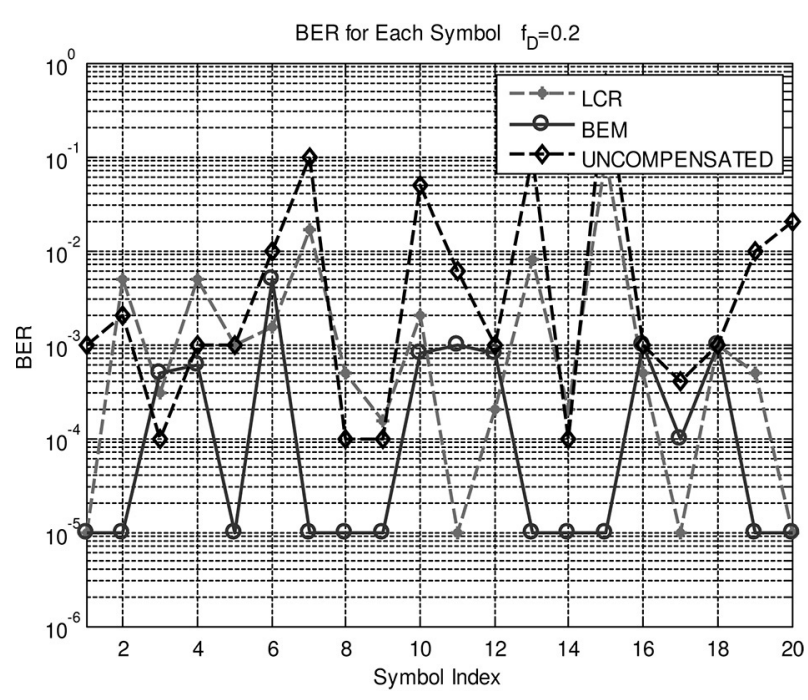

Figure 6. BEM equalizer performance compared with LCR equalizer at $25 \mathrm{~dB}$ of the SNR for $f_{d}=0.2$ in the low speed regime.

pared to the compensated LCR method with the mean BER performance $7.1 \times 10^{-3}$ whereas that of the BEM method is $5.5 \times 10^{-4}$. The mean BER values indicate that the BEM method has a better performance for the $25 \mathrm{~dB}$ of SNR and $f_{d}=0.2$ in the low speed regime, compared to the compensated LCR method. For example, at the 8th symbol index, the BEM method posts the error rate of $10^{-5}$ to outperform the LCR method by $10 \mathrm{~dB}$ and the uncompensated system by $17 \mathrm{~dB}$. Performance evaluations in Fig-

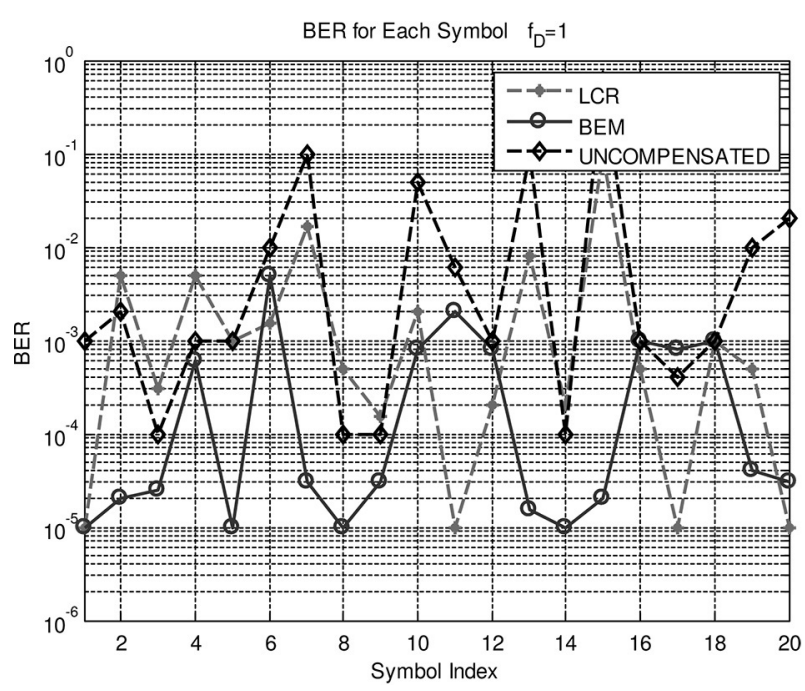

Figure 7. BEM equalizer performance compared with LCR equalizer at $25 \mathrm{~dB}$ of the SNR for $f_{d}=1$ in the high speed regime. ure 6 , have been repeated for moderately high vehicular speed (i.e., $41 \mathrm{~m} / \mathrm{s}$ ) regime as illustrated in Figure 7. For this performance evaluation at $41.7 \mathrm{~m} / \mathrm{s}$ in the highway environment, the findings closely mimic the results found in Figure 6.

The mean BER values for uncompensated system, the compensated LCR method and the BEM method are $4.5 \times 10^{-2}, 7.1 \times 10^{-3}$, and $6.1 \times 10^{-4}$, respectively. The values are consistent with the low speed values where the BEM method performance is still supreme, posting $10.7 \mathrm{~dB}$ and $18.7 \mathrm{~dB}$ above the compensated LCR method and the uncompensated system, respectively. However, the overall performance degradation for the BEM method is observed. This is explained by the increased volatility of the DFS brought about by rapid channel fading due to the high vehicle speed.

Based on the results in Figure 7, the BER performance of the BEM method in comparison with the uncompensated and LCR for a $2 \times 2$ MIMO configurations has been shown in Figure 8.

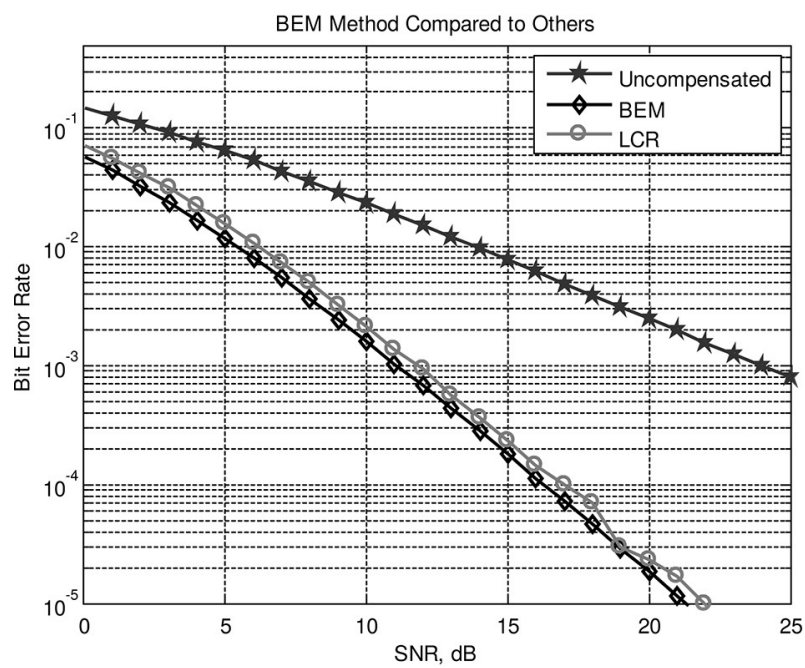

Figure 8. The BEM equalizer (BER) performance under varying SNR conditions.

In Figure 8, it has been noted that the BEM method exhibits some marginal superiority over the LCR across a range of SNR values for the vehicle moving at a speed of $41.7 \mathrm{~m} / \mathrm{s}$ and when a $2 \times 2$ MIMO configuration is taken 
into account. A similar performance evaluation has been conducted for major related DFS estimation and compensation algorithms, namely EVM [2], LMS [3] and LCR [4] for a $2 \times$ 2 MIMO configuration of these schemes in a moderately high speed regime $(\mathrm{v}=41.7 \mathrm{~m} / \mathrm{s})$.

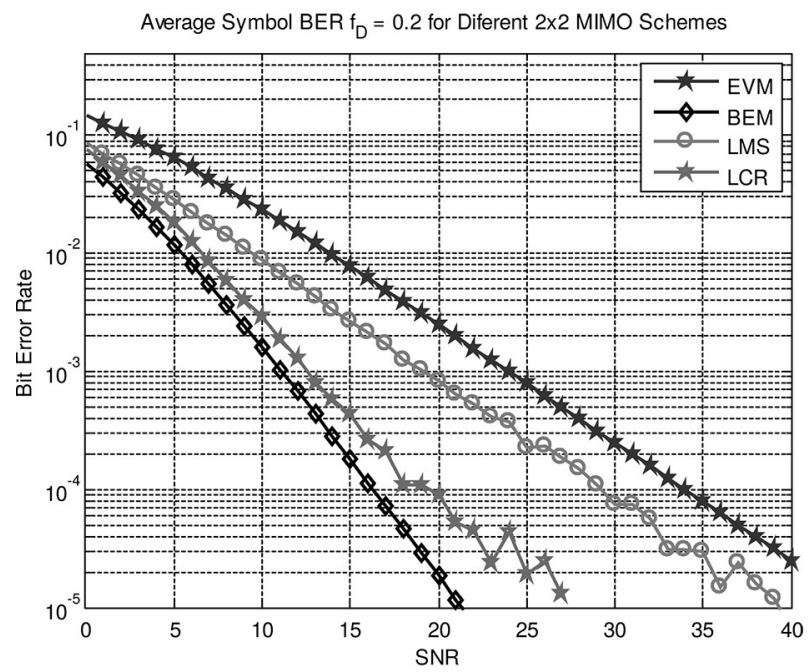

Figure 9. The BEM equalizer (BER) performance under varying SNR conditions.

The findings shown in Figure 9 reveal that the $2 \times 2$ MIMO configurations based BEM is the most superior compared to the other traditional methods. This is attributed to the fact that EVM, LMS and iterative LCR have low multipath channel fading resolving capability at relatively high vehicular speeds. Their OFDM systems are perturbed by DFS such that the inter carrier interference (ICI) is distributed intra and in adjacent subcarriers and the channel matrix becomes heavily banded. However, the BEM method provides a convenient approach to reduce ICI interference by nullifying out-of-band interference. Due to the improved results with both the LCR and BEM based DFS compensated algorithms, Figure 10 aimed at investigating the effects of MIMO configurations on the ergodic capacity performance of the BEM and an iterative LCR.

The results from Figure 10 showed that $N \times N$ BEM outperformed the compensated $N \times N$ iterative LCR model by at least $17 \%$ on average at $14 \mathrm{~dB}$ of SNR under the moderately high speed regime conditions. The performance rises with increasing MIMO order and confirms that

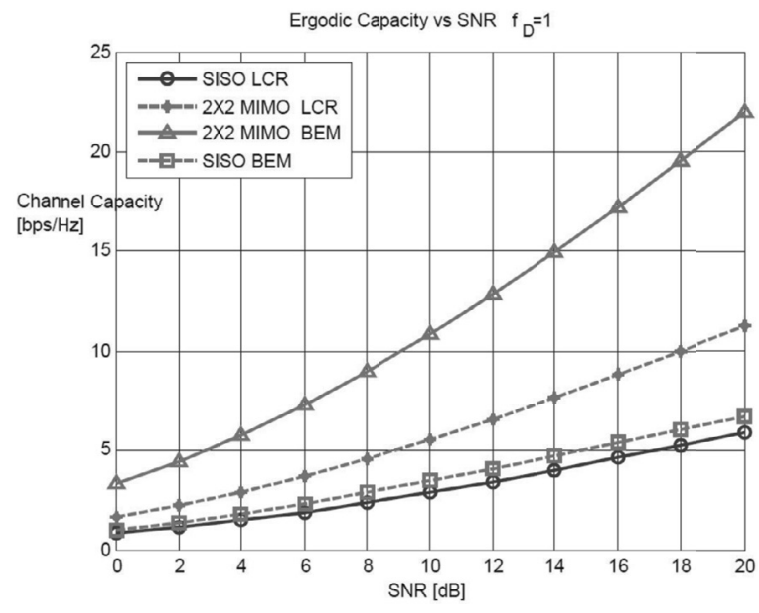

Figure 10. Ergodic capacity of MIMO channel.

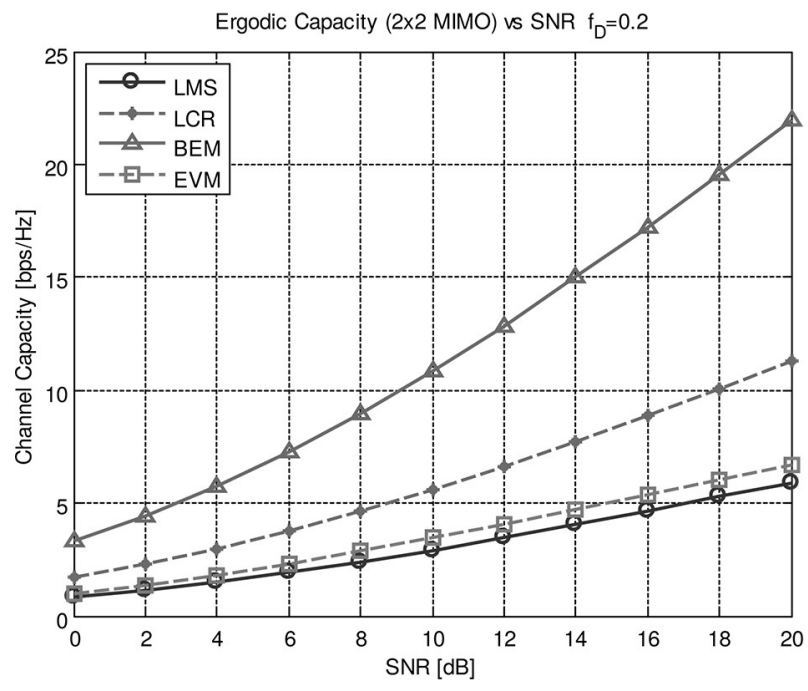

Figure 11. Capacity performance.

future implementation of VANETs will rely on MIMO to support the bulk of ITS applications demanding for high bandwidth. Finally, in Figure 11, capacity performance characteristics of the LMS, LCR, BEM and EVM methods have been presented for $2 \times 2$ MIMO configurations.

The results from Figure 11 showed that the BEM method was the most superior, followed by LCR, EVM and LMS in capacity performance at $f_{d}=0.2$ under the same MIMO configuration. The superiority of the BEM as a DFS estimation and compensation algorithm is justified by its ability to mitigate the effects of multi-path fading, nullify out-of-band interference and simplify computation by reducing the entries to be estimated. Under the BEM meth- 
od, instead of estimating the entire channel matrix entries in $\mathbf{H}_{k}^{D F S}$, only the BEM coefficients extracted from a matrix $\mathbf{B}$ of basis coefficients are estimated.

\section{Validation for Computational Complexity}

As the basis of comparison, we consider an OFDM system with $M=256$ sub carriers. To be able to approximate the TV channel by BEM, we apply Nyquist sampling theorem $Q$ $\geq 2 f_{d}$. In this case we set $f_{d}=1$ such that $Q \geq$ 2, $Q$ being the size of orthonormal basis functions. Furthermore, for the case of comparison, we select $Q=256$ to correspond to the size of the FFT. Assume that for all practical purposes, the maximum transmit-receive antenna pairs are set to be $\Lambda=4$. This implies that for an FIR, the size of the channel taps is $\Lambda^{2}$. Thus, $L+1=\Lambda^{2}$ taps. The TV channel is then characterised with $(L+1)(Q+1)$ coefficients [20]. Consequently, in a single operation with $\Lambda=4,(255+1) \times 16=4096$ coefficients are computed in the BEM-compensated channel as compared to $M \times M=256^{2}=65,536$ points in the uncompensated channel. Hence, the computational complexity in BEM-compensated channel is $\mathrm{O}(M \times N)$ with $N$ representing the number of the OFDM channels, while the computation complexity for the estimated and uncompensated channels is $\mathrm{O}\left(M^{2}\right)$ under high speed MIMO-OFDM VANETS. This implies a larger saving in processing time and memory space.

Table 1. FLOPS count of DFS estimation and compensation existing algorithms.

\begin{tabular}{|c|c|c|}
\hline $\begin{array}{l}\text { The major state of } \\
\text { the art algorithms } \\
\text { for estimating and } \\
\text { compensating } \\
\text { Doppler Shifts (DS) } \\
\end{array}$ & $\begin{array}{l}\text { Determining the Floating point } \\
\text { operations (FLOPS) Computational } \\
\text { Complexity Counts }\end{array}$ & $\begin{array}{l}\text { Steps that cause major increase in the } \\
\text { computational complexity }\end{array}$ \\
\hline $\begin{array}{l}\text { DS estimation based } \\
\text { on Autocorrelation } \\
\text { function }(\mathrm{ACF}) \\
\text { computation }[8]\end{array}$ & $\begin{array}{c}\text { The computation of ACF is a function of } \\
\text { MIMO OFDM systems. The FLOPS count } \\
\text { is mainly viewed as a matrix-matrix product } \\
\text { operation. Suppose MIMO: Matrix is } n \times m \\
\text { and the OFDM: Matrix } n \times p \text { thus, the num- } \\
\text { ber of flops equals to } m p(2 n-1) \text { or } 2 m n p \\
\text { flops for a large value of } n \text {. }\end{array}$ & $\begin{array}{l}\text { The increase in the computational complexity } \\
\text { depends on the multiplicity of MIMOs and } \\
\text { OFDM symbols; the inverse computation of the } \\
\text { Zeroth Order Bessel Function; and the compu- } \\
\text { tational size of the autocorrelation function. }\end{array}$ \\
\hline $\begin{array}{c}\text { Adaptive DS } \\
\text { estimation based on } \\
\text { Level Crossing Rate } \\
\text { (LCR) }[4],[5]\end{array}$ & $\begin{array}{c}\text { For an iterative estimation of the LCR } \\
\text { calculations, vector-vector operations of } \\
\text { number of iterations as well as the vector } \\
\text { of the estimates will constitute the number } \\
\text { of flops. Therefore a scalar multiplication } \\
\text { of two } m \text { and } n \text { vectors would give } n \times m \\
\text { operations. }\end{array}$ & $\begin{array}{l}\text { The increase in the computational complexity } \\
\text { depends on the size of iterations to } \\
\text { convergence; the vector of bias estimates } \\
\text { caused by the noise e.g., AWGN; and the } \\
\text { iterations involved to convergence in } \\
\text { eliminating the bias from the signal. }\end{array}$ \\
\hline $\begin{array}{l}\text { DS estimation based } \\
\text { on Error Vector } \\
\text { Magnitude (EVM) } \\
{[26]}\end{array}$ & $\begin{array}{l}\text { The method employs extended Kalman } \\
\text { filters with a scalar tap size } \alpha \text { and } n \text { states. } \\
\text { Thus the computational complexity is es- } \\
\text { sentially } \alpha \times n \text { flops. }\end{array}$ & $\begin{array}{l}\text { The computational complexity in this method } \\
\text { uses extended Kalman Filter tap sizes, } \\
\text { multiplied by the number of state estimations } \\
\text { being functions of mobile velocity and signal } \\
\text { frequency. The increase in tap sizes, number } \\
\text { of state equations (vectors) would increase the } \\
\text { computational complexity. }\end{array}$ \\
\hline $\begin{array}{l}\text { DS estimation based } \\
\text { on Least Mean } \\
\text { Square (LMS) [3] }\end{array}$ & $\begin{array}{l}\text { In adaptive filtering, the number of flops is } \\
\text { approximated by the scalar weights } \\
\text { multiplied by the } \mathrm{n} \text {-vectors of symbols and } \\
\text { gives } \alpha \times n \text { flops. }\end{array}$ & $\begin{array}{l}\text { The steps that cause increase in the computa- } \\
\text { tional complexity are the determination of the } \\
\text { LMS weights, additions of these weights to the } \\
\text { incoming baseband signal. The received sym- } \\
\text { bols are de-rotated and iterations must converge } \\
\text { linearly with the short training period. }\end{array}$ \\
\hline
\end{tabular}


Furthermore, the computational complexity of the major state of the art algorithms in Floating point operations (FLOPS) is provided in Table 1 for the purpose of comparison. The total flop counts of algorithms involving matrix manipulations are determined by polynomials of the problem dimensions and are often simplified when the lower-order terms are ignored [28].

\section{Conclusion and Future Work}

The paper aimed at improving the time complexity performance of the received signal detection in moderately high speed spatial multiplexed VANET by estimating and compensating Doppler shift. We have modelled a DFS estimator and compensator based on the BEM method. The method has exhibited improved BER performance in comparison with existing methods as a result of better estimated and compensated DFS. This was demonstrated at $15 \mathrm{~dB}$ SNR where the BEM outperformed the uncompensated method by $9 \mathrm{~dB}$ and the LCR method by $1 \mathrm{~dB}$. The features that target multi-path delay spread incorporated in OFDM were exploited to full advantage in the design. It was also noted that the proposed method showed minimal computational complexity under conditions of the high speed and MIMO-OFDM systems. Lastly, the proposed method showed its feasibility for VANET applications that require high capacity channels as well as where wide range of ITS services are amenable. However, an extension of the performance evaluations scope to the non-isotropic scattering fading channels for high vehicular speed (greater than $150 \mathrm{~km} / \mathrm{h}$ ) should be considered. The extension should take into account the von MISES angle of arrival distribution with different Kappa factor to generate directional fading channels.

\section{References}

[1] N. Aboutorab et al., "A New Iterative Doppler-Assisted Channel Estimation Joint with Parallel ICI Cancellation for High-Mobility MIMO-OFDM Systems", IEEE Transactions on Vehicular Technology, vol. 61, no. 4, pp. 1577-1589, 2012. http://dx.doi.org/10.1109/TVT.2012.2189593

[2] F. Nyongesa et al., "Doppler Shift Compensation Schemes in VANETs", Mobile Information Sys- tems, vol. 2015, no. 2015, pp. 1-11, 2015. http://dx.doi.org/10.1155/2015/438159

[3] L. K. Nguyen and R. B. Wells, "Doppler Shift Cancellation using Phasor and Split Phasor LMS Algorithm", in IEEE Military Communications Conference (MILCOM 2008), San Diego, California, 2008, pp. 1-8. http://doi.org/10.1109/MILCOM.2008.4753242

[4] J. Y. Hua et al., "Accurate Estimation of Doppler Shift in Mobile Communications with High Vehicle Speed", International Journal of Communication Systems, vol. 2014, no. 27, pp. 3515-3525, 2014.

http://doi.org/10.1002/dac.2510

[5] J. Y. Hua et al., "An Accurate Scheme for Channel Parameter Estimation in Mobile Propagations", IEICE Transactions on Electronics, vol. E92C, no. 1, pp. 116-120, 2009.

http://doi.org/10.1587/transele.E92.C.116

[6] J. Cai et al., "Doppler Spread Estimation for Mobile OFDM Systems in Rayleigh Fading Channels", IEEE Transactions on Consumer Electronics, vol. 49, pp. 973-977, 2003. http://doi.org/10.1109/TCE.2003.1261183

[7] T. Yucek et al., "Doppler Spread Estimation for Wireless OFDM systems", in IEEE/Sarnoff Symposium on Advances in Wired and Wireless Communication, Princeton, New Jersey, 2005, pp. 233-236.

http://doi.org/10.1109/SARNOF.2005.1426552

[8] J. Mirza et al., "Maximum Doppler Shift Frequency Estimation using Autocorrelation Function for MIMO OFDM Systems", in International Conference on Information and Emerging Technologies, Abbottabad, Pakistan, 2010, pp. 1-6. http://doi.org/10.1109/ICIET.2010.5625717

[9] W. Zhou and W. H. Lam, "A Novel Method of Doppler Shift Estimation for OFDM Systems", in Proceedings of the IEEE Military Communications Conf. MILCOM, Washington DC, 2008, pp.1-6.

http://doi.org/10.1109/MILCOM.2008.4753538

[10] A. E. Abdelkareem et al., "Time Varying Doppler-shift for OFDM-based Shallow Underwater Acoustic Communication Systems", in 8th IEEE International Conference on Mobile AdHoc and Sensor Systems, Valencia, Spain, 2011, pp. 885-891. http://doi.org/10.1109/MASS.2011.105

[11] X. Zhao et al., "Doppler Spread Estimation by Subspace Tracking for OFDM Systems", in IEEE Global Telecommunications Conference (GLOBECOM 2008), New Orleans, 2008, pp. 1-5. http://doi.org/10.1109/GLOCOM.2008.ECP.850

[12] A. Doukas and G. Kalivas, "Doppler Spread Estimation in Frequency Selective Rayleigh Channels for OFDM Systems", in 5th International 
Symposium in Communication Systems, Patras, Greece, 2006, pp. 1-6. http://slideplayer.com/slide/8741133/

[13] A. Mousa and H. Mahmoud, "Reducing ICI Effect in OFDM System using Low-Complexity Kalman Filter Based on Comb-Type Pilots Arrangement", International Journal of Communication Systems, vol. 24, no. 1, pp. 53-61, 2011. http://doi.org/10.1002/dac.1138

[14] Y. S. Cho et al., "MIMO-OFDM Communications with MATLAB", Asia: John Wiley and Sons, 2010. http://doi.org/10.1002/9780470825631

[15] Z. Qin, "Doppler Shift Estimation of MIMO-OFDM Systems based on Autocorrelation Function of Channel Estimate", in Electrical and Computer Engineering, Concordia University: Concordia, 2009.

[16] H. Yi, "Joint Doppler Frequency Shift Compensation and Data Detection Method using a 2-D Unitary ESPRIT Algorithm for SIMO-OFDM Railway Communication Systems", Computer Science, Information Theory. http://arxiv.org/ftp/arxiv/papers/1210/1210.7401.pdf

[17] W.-S. Hou and B.-S. Chen, "ICI Cancellation for OFDM Communication Systems in Time-varying Multipath Fading Channels", IEEE Transactions on Wireless Communications, vol. 4, no. 5, pp. 2100-2110, 2005.

http://dx.doi.org/10.1109/TWC.2005.853837

[18] Z. Tang et al., "Pilot-Assisted Time-Varying OFDM Channel Estimation", in IEEE Int. Conf. on Acoustic, Speech and Signal Processing (ICASSP), Toulouse, France, 2006, pp.1-6. http://dx.doi.org/10.1109/TSP.2007.893198

[19] T. S. Rappaport, "Wireless Communications Principles and Practice", Second ed. 2002, Upper Saddle River, NJ: Prentice Hall.

[20] A. Stamoulis et al., "Intercarrier Interference in MIMO OFDM", IEEE Transactions on Signal Processing, vol. 2002, no. 50, pp. 2451-2464, 2002.

http://dx.doi.org/10.1109/TSP.2002.803347

[21]Z. Tang et al., "Pilot-Assisted Time-Varying Channel Estimation for OFDM Systems", IEEE Transactions on Signal Processing, vol. 55, no. 5, pp. 2226-2238, 2007.

http://dx.doi.org/10.1109/TSP.2007.893198

[22] J. Hao et al., "Joint Channel and Doppler Shift Estimation in TDS-OFDM using Frequency Sensitive Set", in IEEE Proceedings: Broadband multimedia systems and broadcasting (BMSB), Beijing, China, 2014, pp. 1-6. http://dx.doi.org/10.1109/BMSB.2014.6873568

[23] A. Ligata et al., "A Performance Analysis of MIMO-OFDM/TDM in a Peak-limited Multi-path Fading Channel", in IEEE Asia-Pacific Confer- ence on Communications (APCC), Kota Kinabalu Sabah, Malaysia, 2011.

http://dx.doi.org/10.1109/APCC.2011.6152875

[24] H. Bolcskei, "MIMO-OFDM Wireless Systems: Basics, Perspectives, and Challenges", IEEE Wireless Communications, vol. 13, no. 4, pp. 31-37, 2006. http://dx.doi.org/10.1109/MWC.2006.1678163

[25] A. El-Keyi et al., "MIMO VANETS: Research Challenges and Opportunities", in IEEE Proc. Int. Conf. On Computing, Networking and Communications, Maui, Hawaii, USA, 2012, pp.1-6. http://dx.doi.org/10.1109/ICCNC.2012.6167507

[26] B. Kusy et al., "Tracking Mobile Nodes using RF Doppler Shifts", in IEEE SenSys'07, Sydney, Australia, Nov. 6-9, 2007. http://dx.doi.org/10.1145/1322263.1322267

[27] T.A Lemahewa et al., "Space-Time Cross Correlation and Space-Frequency Cross Spectrum in Non-Isotropic Scattering Environments", 2006 IEEE International Conference on Acoustics Speech and Signal Processing, Toulouse, France, May 14-19, 2006.

https://dblp.org/pers/hd/1/Lamahewa:Tharaka_A=

[28] R. P. Brent and P. Zimmermann, "Modern Computer Arithmetic", Cambridge University Press, 2010.

Received: December 2017 Revised: June 2018 Accepted: July 2018

Contact addresses:

Ferdinand C. Nyongesa

Masinde Muliro University of Science and Technology

Kakamega, Kenya e-mail: fnyongesa@mmust.ac.ke

Thomas O. Olwal Department of Electrical Engineering Tshwane University of Technology Pretoria, South Africa e-mail: olwalto@tut.ac.za 
Ferdinand C. Nyongesa received his BSc degree in Physics in 1983 from the University Of Nairobi, Kenya, a graduate Diploma in Electrical and Communication Engineering from the University of Bradford in the UK in 1987 and an MSc in Communication Engineering and Digital Electronics in 1990 from the same University. He received a Doctor of Technology title in Electrical Engineering from the Tshwane University of Technology, South Africa in 2017. He lectures at Masinde Muliro University of Science and Technology and with extensive industrial experience blended with academia throughout his career life. His research interests are in the design and applications of the Intelligent Transportation systems, the VANETS, AD-HOC Networks and Advanced Wireless Sensor Networks. He has published several articles in peer reviewed journals and conferences.

Thomas O. Olwal a senior member of IEEE, received the PhD in Computer Science from the University of Paris-EST, France, in 2010, and the Doctor of Technology title in Electrical Engineering from Tshwane University of Technology (TUT), South Africa, in 2011. He is a registered professional engineer and currently works at TUT as an Associate Professor. His research interests include analysis and design of the spectrum, energy-efficient radio resource management, Internet of Things, advanced wireless sensor networks, SDN, Cognitive Radios, TV White spaces and Intelligent Networks. He has published over 115 technical and scientific research outputs in peer reviewed accredited journal articles, book chapters and conference papers. He also serves as a reviewer in a number of ACM/IEEE conferences and journals. 